\title{
The Prism-Pair: Simple Dispersion Compensation and Spectral Shap- ing of Ultrashort Pulses
}

\author{
Yaakov Shaked', Shai Yefet ${ }^{2}$ and Avi Pe'er ${ }^{1 *}$ \\ ${ }^{1}$ Department of physics and BINA Center for Nano-technology, Bar-Ilan University, Ramat-Gan 52900, Israel \\ ${ }^{2}$ Ophthalmology division, Lumenis LTD, Yokneam 2069204, Israel
}

\begin{abstract}
A simple and intuitive formulation is reviewed for the Brewster prism-pair - A most common component in spectroscopy-oriented experiments using ultrashort pulses. This review aims to provide students and beginners in the field of spectroscopy with a unified description of a major experimental component. The total spectral phase experienced by a broadband light field is calculated after passing through a pair of Brewster-cut prisms, demonstrating the flexibility of the prism pair to provide tuned, low-loss control of the dispersion and spectral phase experienced by ultrashort pulses.
\end{abstract}

\section{Introduction}

The generation of ultrashort pulses [1,2] revolutionized the field of molecular spectroscopy [3]. Not only that ultrashort pulses enable to observe molecular motion in time [4-6], but they allow also manipulation of the molecular dynamics [7-9] by controlling the spectral properties of the pulses. For this end, many types of pulseshaping techniques and configurations are common in spectroscopyoriented experiments. When complete control of the spectral amplitude and phase is required, a general Fourier-domain pulseshaper [10] can be used, which provides independent control of both phase and amplitude for each frequency component of the light (e.g. with a spatial light modulator [11,12] or deformable mirrors [13]). However, most applications require only much simpler control of the group delay dispersion (GDD) and higher order dispersion to compensate for the dispersive effect experienced by an ultrashort pulse when passing through optical media and setups. For these applications, the high internal loss and technical complexity of a general pulse-shaper are a burden, and simpler configurations are commonly used, such as the grating-pair [14], chirped mirrors [15] or the Brewster prism-pair [16] - the subject of this mini-review. Prismpairs can provide tuned compensation with ultra-low loss of up to two orders of dispersion (GDD and sometimes an additional higher order), along with simple amplitude control using a slit or transmission mask in the dispersive arm. Due to the low loss of the prism-pair, it is often the main 'tool of choice' for intra-cavity applications [17-19], low light level spectroscopy [20-22], and quantum optics experiments [23-27]. This mini-review presents a simple and intuitive formulation of the total wavelength-dependent phase accumulated by light passing through a prism-pair, and demonstrates how this major component is used for tuned, precise control of dispersion and spectral phase.

\section{A Single Prism}

The analysis starts by reviewing the geometry of a single prism. When a ray passes through a prism at minimum deviation, the angles of refraction through the prism are symmetric [28], as illustrated in figure 1 , resulting in:

$$
\begin{aligned}
& \theta_{1}=\theta_{4} \\
& \theta_{2}=\theta_{3}=\frac{\alpha}{2}
\end{aligned}
$$

where $\alpha$ is the apex angle of the prism. If the entrance angle matches with the Brewster angle $\theta_{B}$ (for a certain wavelength $\lambda_{0}$ ), the refraction angles obey

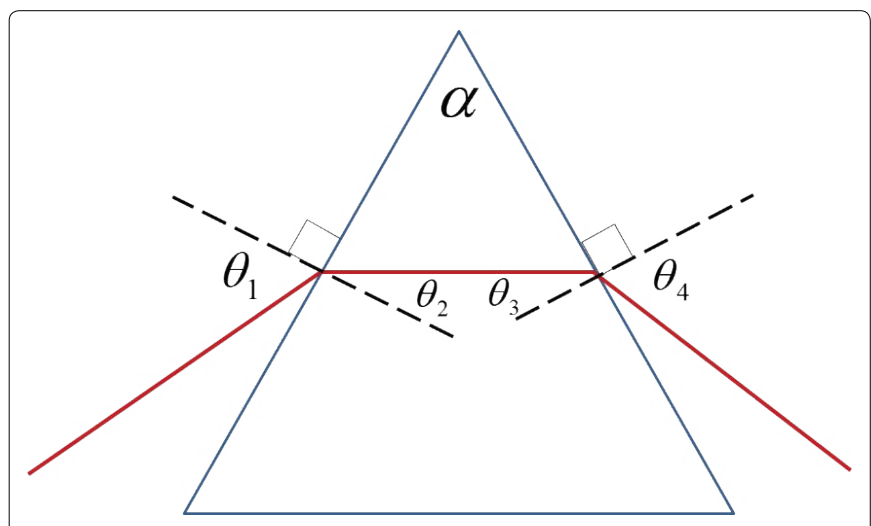

Figure 1: Symmetry relations between the refraction angles in a prism. At minimum deviation, the entrance and exit angles are equal and the ray propagates through the prism parallel to its base.

*Corresponding author: Avi Pe'er, Physics department and BINA center for nanotechnology, Bar llan University, Ramat Gan 52900, Israel, E-mail: avi.peer@biu.ac.il

Received: January 27, 2016: Accepted: April 04, 2016: Published: April 07, 2016

Copyright: $\odot 2016$ Shaked $Y$, et al. This is an open-access article distributed under the terms of the Creative Commons Attribution License, which permits unrestricted use, distribution and reproduction in any medium, provided the original author and source are credited. 


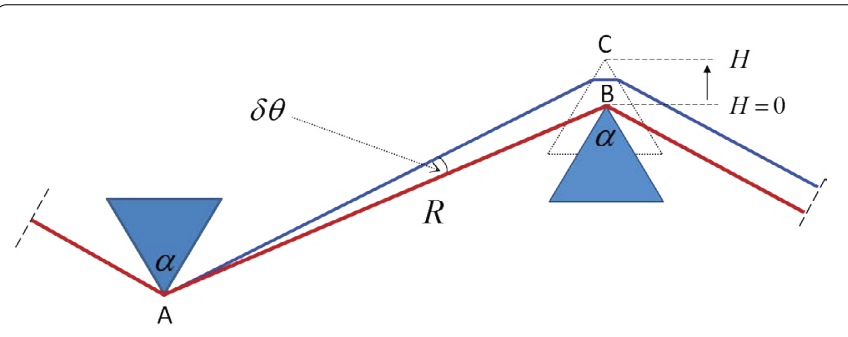

Figure 2: Geometry of a prism-pair, defined using two degrees of freedom: the separation between the prisms $\mathrm{R}$ and the prism insertion $\mathrm{H}$.

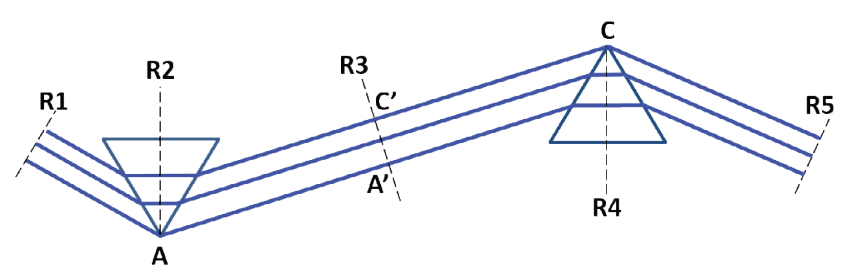

Figure 3: Different wavefronts (black dashed lines) of equal phase in a plane wave beam passing through a prism pair.

$$
\begin{aligned}
& \theta_{B}=\theta_{1}=\theta_{4} \\
& \theta_{B}+\theta_{2}=\frac{\pi}{2}
\end{aligned}
$$

and the relation between the apex angle $\alpha$ and Brewster angle can be expressed as

$$
\alpha=2 \theta_{2}=2\left(\frac{\pi}{2}-\theta_{B}\right)=\pi-2 \theta_{B}
$$

\section{The Optical Path through a Prism-Pair}

Our aim now is to calculate the total optical path (and phase) through a prism-pair, which will allow to derive the dispersion experienced by the passing optical pulse. The coordinate system used for the prism-pair has two degrees of freedom (Figure 2): $\mathrm{R}$ is the separation between the prisms (segment $\mathrm{AB}$ ), and $\mathrm{H}$ is the penetration of the prism into the beam (segment $\mathrm{BC}$ ). The red line represents a Brewster ray (at the design wavelength $\lambda_{0}$ ) that enters and exits both prisms at Brewster angle $\theta_{B}$. The blue line is the deviated ray at $\lambda \neq \lambda_{0}$, which deviates from the Brewster ray by an angle $\delta \theta$ due to the prism dispersion.

\section{Wavefront Calculation}

To calculate the phase accumulated by an arbitrary wavelength component $\lambda$ (blue line) through the prism-pair, one usually calculates the optical path of the deviated beam along a continuous ray, starting from point $\mathrm{A}$, through multiple refractions in the $2^{\text {nd }}$ prism until a final reference plane perpendicular to the beam, located after the $2^{\text {nd }}$ prism where all the colors are parallel $[29,30]$. This method results in somewhat complicated expressions for the optical path, leaving little room for intuition. An elegant alternative (outlined in Figure 3) that avoids calculations of multiple refractions is to use the concept of wavefronts for a non-continuous ray. This concept was originally presented in [31] and calculated to include only the prisms separation $\mathrm{R}$. The following generalizes the calculation to include also the prism insertion $\mathrm{H}$.

Each of the rays within a plane-wave beam experiences the same optical path (and phase) through the prism-pair, as illustrated in figure 3, starting from reference plane R1 to R5 (all the rays have the same wavelength $\lambda_{0}$ ). Note that the optical phase is constant across each of the reference planes (dashed black lines), since each of them coincides with the wavefront perpendicular to the beam. The above argument is true not only for the Brewster ray $\lambda_{0}$, but for any other

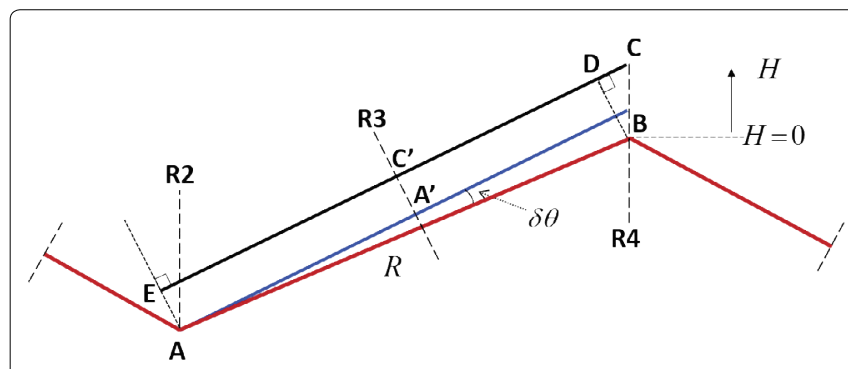

Figure 4: Complete optical path through a prism-pair. Following a noncontinuous ray by using the concept of wavefronts, the optical path through the entire system can be reduced to the segment EC.

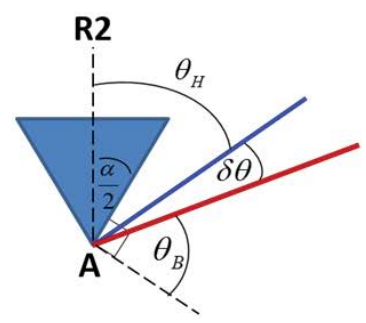

Figure 5: Definition of the exit angles in the $1^{\text {st }}$ prism.

wavelength $\lambda$. Since the wavefront $\mathrm{R} 1$ is common for all wavelengths, shifting its position only results in a simple optical delay, hence, the position of R1 can be chosen freely to pass through the point A. The same argument applies also to R5, which can be chosen to pass through point $\mathrm{C}$.

Consequently, for calculating the geometric dispersion, it is sufficient if the optical path through the entire prism-pair is reduced to the segment:

$$
P=A A^{\prime}+C C^{\prime}
$$

Note that although this path is composed of free-space rays without refractions, it already takes into account refraction of the planar beam in the material. As illustrated in figure 4 (which shows the rays without the prisms for clarity), the optical path in Eq. 4 is identical to the segment $E C$, where the point $C$ is the vertex of the $2^{\text {nd }}$ prism. The conclusion is that the optical path of the deviated ray through the entire prism pair is simply given by:

$$
P=E C=E D+D C
$$

where $E D=R \cos \delta \theta$, and $D C=H \cos \theta_{H}\left(\theta_{H}=\angle D C B\right)$.

Since $E C$ is parallel to $A A^{\prime}$ and $\mathrm{R} 2$ is parallel to $\mathrm{R} 4$, one can see that $\theta_{H}$ is also the angle between $\mathrm{R} 2$ and the deviated ray (blue line) in the $1^{\text {st }}$ prism, as illustrated in figure 5 :

$$
\theta_{H}=\frac{\alpha}{2}+\frac{\pi}{2}-\theta_{B}-\delta \theta
$$

Substituting Eq. 3 into Eq. 6 obtains:

$$
\theta_{H}=\pi-2 \theta_{B}-\delta \theta=\alpha-\delta \theta,
$$

indicating that $D C=H \cos (\alpha-\delta \theta)=H \cos \alpha \cos \delta \theta+H \sin 2 \theta_{B} \sin \delta \theta$.

By substituting the above relations into Eq. 5, the total optical path through the prism-pair is:

$$
P=(R+H \cos \alpha) \cos \delta \theta+H \sin 2 \theta_{B} \sin \delta \theta
$$

and the optical phase experienced by frequency $\omega$ is $\varphi=\frac{\omega}{c} P$

Note that Eq. 8 was a generalization of the method presented in [31] in which only the special case of $H=0$ is presented.

The expressions in equations [8] and [9] for the optical path $P$ 


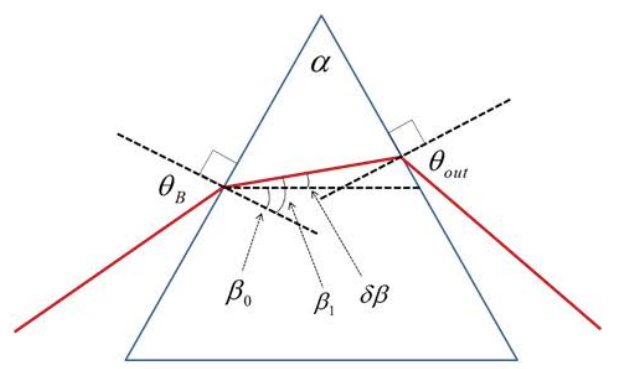

Figure 6: Definition of the refraction angles for small angle approximation of the angular dispersion.

and optical phase $\varphi$ are exact and can be easily used for numerical calculation of the spectral phase $\varphi(\omega)$ and its frequency derivatives $G D=d \varphi / d \omega, G D D=d^{2} \varphi / d \omega^{2}$ and higher order dispersion terms. The refraction angle $\delta \theta$ for each wavelength/frequency can be calculated according to Snell's law by using the prism's apex angle and the Sellmeier formula for either $n(\lambda)$ or $n(\omega)$.

\section{Approximation of the Prism Angular Dispersion}

Although Eq. 8 and Eq. 9 already allow complete calculation of the dispersion properties, much intuition can be gained by further developing the expressions with the assumption that the angle $\delta \theta(\lambda)$ is small. Replacing $\cos \delta \theta \approx 1-\delta \theta^{2} / 2$ and $\sin \delta \theta \approx \delta \theta$ yields:

$$
P \approx(R+H \cos \alpha) \cdot\left(1-\delta \theta^{2} / 2\right)+\left(H \sin 2 \theta_{B}\right) \cdot \delta \theta
$$

The angle $\delta \theta$ can now be expressed using Snell's law assuming small angles, such that: $\cos \delta \theta \approx 1$ and $\sin \delta \theta \approx \delta \theta$. It is assumed that the beam enters the 1st prism at the minimum deviation angle for a certain wavelength $\lambda_{0}$; that the entrance angle matches the Brewster angle $\theta_{B}$ for $\lambda_{0}$; and that the prism refractive index for $\lambda_{0}$ is $n\left(\lambda_{0}\right)=n_{0}$. The refractive index for the deviated beam $\left(\lambda \neq \lambda_{0}\right)$ is: $n=n_{0}+\delta n(\lambda)$.

The angle of refraction inside the prism for the Brewster beam is $\beta_{0}$ (equal at both faces of the prism). As illustrated in figure 6 , the angle of refraction for the deviated beam inside the prism is $\beta_{1}=\beta_{0}+\delta \beta$ . It is easy to show that the deviated beam will hit the exit face of the prism at an angle of $\beta_{2}=\beta_{0}-\delta \beta$. The exit angle for the deviated beam will be $\theta_{\text {out }}=\theta_{B}+\delta \theta$. In addition, since at Brewster angle $\tan \theta_{B}=n_{0}$ and $\theta_{B}=\pi / 2-\beta_{0}$, the following relations hold: $\sin \theta_{B}=\cos \beta_{0}$ and $\cos \theta_{B}=\sin \beta_{0}$.

Following Snell's law from the entrance face to the exit, an approximation of the deviation angle $\delta \theta$ can be obtained. At the entrance:

$$
\sin \theta_{B}=n \sin \beta_{1} \quad \text { [11] }
$$

Expanding the right hand side yields

$$
\begin{aligned}
n \sin \beta_{1} & =\left(n_{0}+\delta n\right) \sin \left(\beta_{0}+\delta \beta\right) \\
& =\left(n_{0}+\delta n\right)\left(\sin \beta_{0} \cos \delta \beta+\cos \beta_{0} \sin \delta \beta\right) \\
& \approx\left(n_{0}+\delta n\right)\left(\sin \beta_{0}+\delta \beta \cos \beta_{0}\right) \\
& =n_{0} \sin \beta_{0}+\delta n \sin \beta_{0}+\delta \beta n_{0} \cos \beta_{0} \\
& =n_{0} \cos \theta_{B}+\delta n \cos \theta_{B}+\delta \beta n_{0} \sin \theta_{B}
\end{aligned}
$$

Substituting into Eq. 11 and dividing by $\sin \theta_{B}$ (and noting that $\tan \theta_{B}=n_{0}$ ) provides

$$
\begin{aligned}
& 1+\frac{\delta n}{n_{0}}+n_{0} \delta \beta=1 \\
& n_{0}^{2} \delta \beta=-\delta n
\end{aligned}
$$

Following similar logic at the exit face of the prism, obtains

$$
n \sin \beta_{2}=\sin \theta_{\text {out }}
$$

Expanding both sides of Eq. 14 yields

$$
\begin{aligned}
n \sin \beta_{2} & =\left(n_{0}+\delta n\right) \sin \left(\beta_{0}-\delta \beta\right) \\
& \approx n_{0} \sin \beta_{0}+\delta n \sin \beta_{0}-\delta \beta n_{0} \cos \beta_{0} \\
& =n_{0} \cos \theta_{B}+\delta n \cos \theta_{B}-\delta \beta n_{0} \sin \theta_{B} \\
\sin \theta_{\text {out }} & =\sin \left(\theta_{B}+\delta \theta\right)=\sin \theta_{B} \cos \delta \theta+\cos \theta_{B} \sin \delta \theta \\
& \approx \sin \theta_{B}+\delta \theta \cos \theta_{B}
\end{aligned}
$$

Equating both sides yields

$$
n_{0} \cos \theta_{B}+\delta n \cos \theta_{B}-\delta \beta n_{0} \sin \theta_{B}=\sin \theta_{B}+\cos \theta_{B} \delta \theta
$$

Dividing Eq. 16 by $\sin \theta_{B}$ provides

$$
\begin{aligned}
& 1+\frac{\delta n}{n_{0}}-n_{0} \delta \beta=1+\frac{\delta \theta}{n_{0}} \\
& \delta n-n_{0}^{2} \delta \beta=\delta \theta
\end{aligned}
$$

Substituting Eq. 13 into Eq. 17 yields

$$
\delta \theta=2 \delta n=2\left(n-n_{0}\right)
$$

Finally, Eq. 18 can be substituted into Eq. 8 and Eq. 9 to retrieve the total wavelength dependent phase through the prism-pair. Since the wavelength/frequency dependence is now only in $\delta n(\lambda)$, a simple expression for the $G D D=d^{2} \varphi / d \omega^{2}$ can be derived:

$$
\frac{d^{2} \varphi}{d \omega^{2}}=\frac{2}{c}(B-2 A \delta n)\left(2 \frac{d n}{d \omega}+\omega \frac{d^{2} n}{d \omega^{2}}\right)-\frac{4 \omega A}{c}\left(\frac{d n}{d \omega}\right)^{2}
$$

where $A=R+H \cos \alpha$ and $B=H \sin \theta_{B}$. The wavelength dependent refractive index of the prisms $n$ can be obtained from the Sellmeier formula.

The exact spectral phase and any of its derivatives $\left(d^{m} \varphi / d \omega^{m}\right)$ can be easily calculated using the chain rule [32]:

$$
\begin{aligned}
& \frac{d n}{d \omega}=-\frac{\lambda^{2}}{2 \pi c} \frac{d n}{d \lambda} \\
& \frac{d^{2} n}{d \omega^{2}}=\frac{\lambda^{3}}{2 \pi^{2} c^{2}}\left(\frac{d n}{d \lambda}+\frac{\lambda}{2} \frac{d^{2} n}{d \lambda^{2}}\right)
\end{aligned}
$$

For typical optical bandwidths it is safe to assume that $B \gg 2 A \delta n$ and that $R \gg H$, since $R \sim 10-50 \mathrm{~cm}$ and $H \sim 10 \mathrm{~mm}$ are common values. Thus, it is intuitive to think of $A$ as the distance between the prisms ( $A \approx R$ ), and of $B$ as the propagation distance inside the prisms. Further approximation can be made since for most practical optical materials $2 \cdot(d n / d \omega) \ll \omega \cdot\left(d^{2} n / d \omega^{2}\right)$. Hence, the total GDD of the prism pair is the sum of two parts: the accumulated GDD from the material dispersion due to propagation through the prisms and an additional negative GDD resulting from the geometric dispersion from the path between the prisms:

$$
\frac{d^{2} \varphi}{d \omega^{2}} \approx \frac{2 B \omega}{c} \frac{d^{2} n}{d \omega^{2}}-\frac{4 A \omega}{c}\left(\frac{d n}{d \omega}\right)^{2}
$$

The geometric dispersion in the second part attributes always a negative GDD for any material (independent of the sign of $d n / d \omega$ ), whereas the first part depends on the material dispersion, which may be either positive (as is usually the case in the visible or NIR range), or negative (as is usually the case for the IR in most materials). Hence, a simple prism-pair offers tunable GDD, both negative and positive, for visible and NIR wavelengths, but for the IR range it will commonly provide only negative GDD. Tuned positive dispersion in the IR range can still be obtained by inserting a 1x1_telescope between the prisms that can flip the sign of the geometric dispersion by imaging the first prism beyond the 2nd prism, effectively generating a "negative distance" $R$ between the prisms [23].

\section{Summary}

The performance of the Brewster prism-pair was reviewed - a common major component of ultrafast spectroscopy apparati. The total spectral phase was calculated as accumulated by broadband light 
in passage through the prism-pair. Following a simple and intuitive approach using the concept of wavefronts, it was showed how the total optical path through a prism pair can be reduced to the path of a non-continuous beam, hence avoiding unnecessary computations of multiple refractions inside the prisms. Finally, the total phase and any of its derivatives can be easily calculated. Specifically, by careful choice of the two degrees of freedom of the prism pair $\mathrm{R}$ and $\mathrm{H}$, it is possible to compensate two orders of dispersion simultaneously (e.g. $d^{2} \varphi / d \omega^{2}$ and $\left.d^{3} \varphi / d \omega^{3}\right)$, which is crucial when using ultra-broadband light to maintain high temporal resolution [23].

\section{References}

1. Hargrove LE, R. Fork L, Pollack MA (1964) Locking of hene laser modes induced by synchronous intracavity modulation. Appl Phys Lett 5: 4-5.

2. Spence DE, Kean PN, Sibbett W (1991) 60-fsec pulse generation from a selfmode-locked ti: sapphire laser. Opt Lett 16: 42-44.

3. Zewail AH (2000) Femtochemistry: Atomic-scale dynamics of the chemical bond. J Phys Chem A 104: 56605694.

4. Tannor DJ, Kosloff R, Rice SA (1986) Coherent pulse sequenceinduced control of selectivity of reactions: Exact quantum mechanicalcalculations. J chem phys 85: 5805-5820.

5. Shapiro M, Brumer P (1996) Coherent control of collisional events: Bimolecular reactive scattering. Phys rev lett 77: 2574

6. Hansch TW (2006) Nobel lecture: passion for precision. Reviews of Modern Physics 78: 1297.

7. Dhar L, Rogers JA, Nelson KA (1994) Time-resolved vibrational spectroscopy in the impulsive limit. Chem Rev 94: 157-193.

8. Assion A, Baumert T, Bergt M, Brixner T, Kiefer B, et al. (1998) Control of chemical reactions by feedback optimized phase-shaped femtosecond laser pulses. Science 282: 919-922.

9. Aharonovich I, Peer A (2016) Coherent amplification of ultrafast molecular dynamics in an optical oscillator. Phys rev lett 116: 073603.

10. Weiner AM, Heritage JP, Kirschner EM (1988) High-resolution femtosecond pulse shaping. J Opt Soc Am B 5: 1563-1572.

11. Dayan B, Peer A, Friesem AA, Silberberg $Y(2005)$ Nonlinear interactions with an ultrahigh flux of broadband entangled photons. Phys rev lett 94: 043602.

12. Weiner AM Ultrafast (2011) optical pulse shaping: A tutorial review. Opt Comm 284: 3669-3692.

13. Weinacht T, Bartels R, Backus S, Bucksbaum P, Pearson B, et al. (2001) Coherent learning control of vibrational motion in room temperature molecular gases. Chem Phys Lett 344: 333-338.

14. Oron D, Dudovich N, Yelin D, Silberberg Y (2002) Narrow-band coherent antistokes Raman signals from broad-band pulses. Phys Rev Lett 88: 063004
15. Vered RZ, Rosenbluh M, Pe'er A (2012) Two-photon correlation of broadbandamplified spontaneous four-wave mixing. Phys Rev A86: 043837.

16. Gershgoren E, Bartels RA, Fourkas JT, Tobey R, Murnane MM, et al. (2003) Simplified setup for high-resolution spectroscopy that uses ultrashort pulses. Opt Lett 28: 361-363.

17. Arissian $L$ and Diels JC (2007) Carrier to envelope and dispersion control in a cavity with prism pairs. Phys Rev A75: 013814.

18. Fork RL, Cruz CB, Becker P, Shank CV (1987) Compression of optical pulses to six femtoseconds by using cubic phase compensation. Opt lett 12: 483-485.

19. Spence D, Sibbett W (1991) Femtosecond pulse generation by a dispersioncompensated, coupled-cavity, mode-locked ti: sapphire laser. JOSA B 8: 2053-2060.

20. Ogilvie JP, Debarre D, Solinas X, Martin JL, Beaurepaire E, et al. (2006) Use of coherent control for selective two-photon fluorescence microscopy in live organisms. Optics express 14: 759-766.

21. Entenberg D, Wyckoff J, Gligorijevic B, Roussos ET, Verkhusha VV, et al. (2011) Setup and use of a two laser multiphoton microscope for multichannel intravital fluorescence imaging. Nat protoc 6: 1500-1520.

22. Kobayashi T, Yoshizawa M, Stamm U, Taiji M, Hasegawa M (1990) Relaxation dynamics of photo-excitations in polydiacetylenes and polythiophene. J Opt Soc Am B 7: 1558-1578.

23. Shaked Y, Yefet S, Geller T, Peer A (2015) Octave-spanning spectral phase control for single-cycle bi-photons. New Journal of Physics 17: 073024.

24. Shaked Y, Pomerantz R, Vered RZ, Pe'er A (2014). Observing the nonclassical nature of ultra-broadband bi-photons at ultrafast speed. New Journal of Physics 16: 053012.

25. Sensarn S, Yin GY, Harris SE (2010) Generation and compression of chirped bi-photons. Phys Rev Lett 104: 253602.

26. Peer A, Silberberg Y, Dayan B, Friesem AA (2006) Design of a high power continuous source of broadband down-converted light. Phys Rev A 74: 053805.

27. Bessire B, Bernhard C, Feurer T, Stefanov A (2014) Versatile shaper-assisted discretization of energy-time entangled photons. New Journal of Physics 16: 033017.

28. Hecht E (1987) Optics (Addison Wesley).

29. Yang S, Lee K, Xu Z, Zhang X, Xu X (2001) An accurate method to calculate the negative dispersion generated by prism pairs. Optics and Lasers in Engineering 36: 381-387.

30. Arissian L, Diels JC (2007) Carrier to envelope and dispersion control in a cavity with prism pairs. Phys Rev A75: 013814.

31. Fork RL, Martinez E, Gordon JP (1984) Negative dispersion using pairs of prisms. Opt Lett 9: 150-152.

32. Cojocaru E (2003) Analytic expressions for the fourth- and the fifth-order dispersions of crossed prisms pairs. Appl Opt 42: 6910-6914. 